\title{
Exploration of Planetary Terrains with a Legged Robot as a Scout Adjunct to a Rover
}

\author{
Silvano Colombano" and Frank Kirchner ${ }^{\dagger}$ \\ NASA-Ames Moffett Field CA 94035 and University of Bremen, 28359 Bremen, Germany \\ Dirk Spenneberg ${ }^{\ddagger}$ \\ University of Bremen, 28359 Bremen, Germany \\ and \\ James Hanratty \\ NASA-Ames Moffett Field CA 9403
}

\begin{abstract}
The Scorpion robot is an innovative, biologically inspired 8-legged walking robot. It currently runs a novel approach to control, which utilizes a central pattern generator (CPG) and local reflex action for each leg. From this starting point we are proposing to both extend the system's individual capabilities and its capacity to function as a "scout" cooperating with a larger wheeled rover. For this purpose we propose to develop a distributed system architecture that extends the system's capabilities both in the direction of high level planning and execution in collaboration with a rover, and in the direction of force-feedback based low level behaviors that will greatly enhance its ability to walk and climb in rough varied terrains. The final test of this improved ability will be a rappelling experiment where the Scorpion explores a steep cliff side in cooperation with a rover that serves as both anchor and planner/executive.
\end{abstract}

\section{Introduction}

N

ASA needs autonomous robotic exploration of difficult (rough and/or steep) scientifically interesting planetary terrains. We have been proposing to utilize a legged robot ("Scorpion", Kirchner et al. ${ }^{1}$ ) as an adjunct scout to a rover for access to difficult - scientifically interesting - terrains (rocky areas, slopes, cliffs). One test mission scenario involves the Ames rover platform , $\mathrm{K} 9^{\text {"k }}$ and $\mathrm{Scorpion}$ acting together to explore a steep cliff, with the Scorpion robot rappelling down using the $\mathrm{K} 9$ as an anchor as well as mission planner and executive. Cooperation concepts, including wheeled rappelling robots have been proposed before ${ }^{2}$. Our focus is on testing the combined advantages of a wheeled vehicle with a legged scout as well as the advantages of merging high level planning and execution with biologically inspired, behavior based robotics. We propose to use the 8-legged, multifunctional autonomous robot platform Scorpion (previously developed under DARPA contract) that is currently capable of walking on different terrains (rocks, sand, grass,...) and perceiving its environment while modifying its behavioral pattern accordingly.

These capabilites would be extended to enable the Scorpion to communicate and cooperate with a partner robot and to climb over rocks, rubble piles, and objects with structural features. This will be done in the context of exploration of rough terrains in the neighborhood of the rover, but inaccessible to it, culminating in the added capability of rappelling down a steep cliff for both vertical and horizontal terrain observation. (fig.2). Development plans for the Scorpion include the ability to grasp and manipulate objects.

\footnotetext{
- Computer Scientist, Intelligent Robotics Group, MS 269-2, AIAA Senior Member.

${ }^{\dagger}$ Professor, Dept. Computer Science and Mathematics, MZH 5080 Bibliothekstr. 1

${ }^{\ddagger}$ Senior Scientist, Dept. Computer Science and Mathematics, MZH 5080 Bibliothekstr. 1

${ }^{\S}$ Electronics Engineer, Projects Division, MS213-2
} 

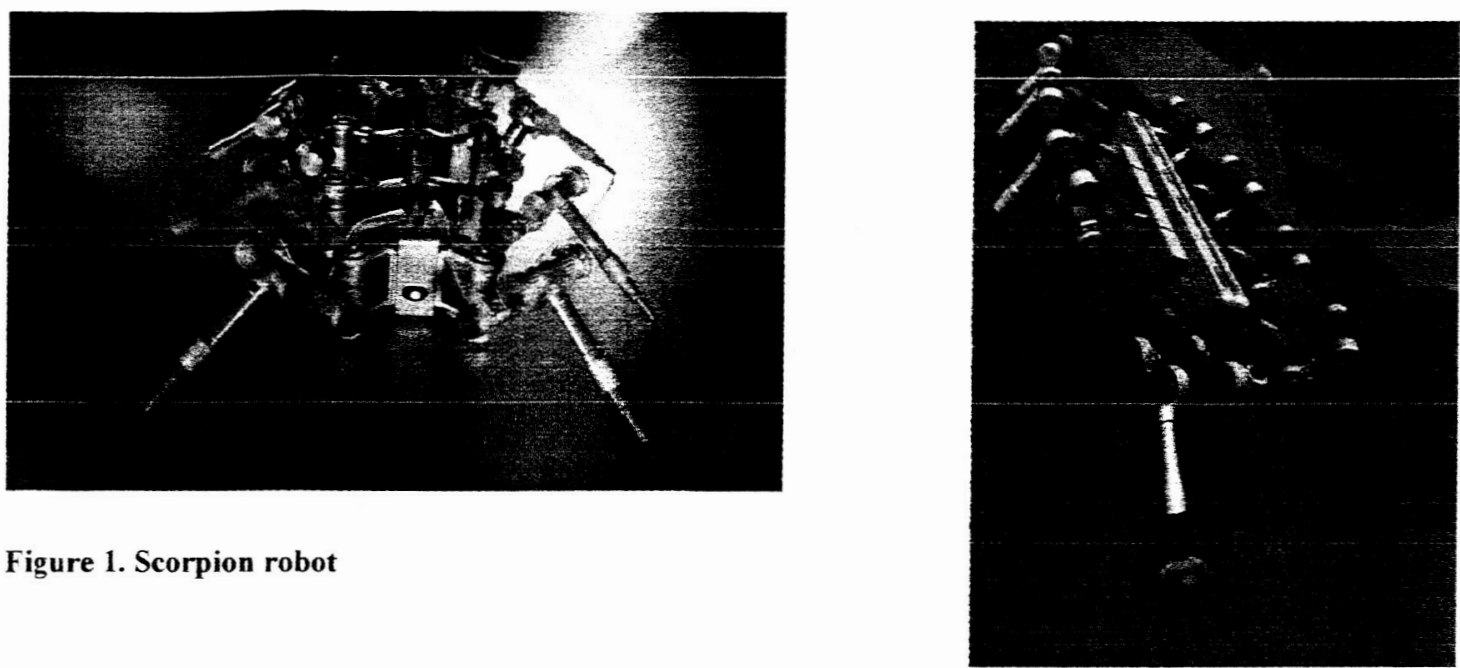

Figure 2. Concept design:

Scorpion rappelling down a cliffside.

In this paper we will describe the architecture of the Scorpion system, its present and future capabilities, and some issues involving the cooperation of rover class robots with legged robots for rough terrain exploration.

\section{The Scorpion System}

The robot we plan to use for the exploration will be a modified version of a system developed in the lab of Prof. Frank Kirchner, presently at the University of Bremen in a previously DARPA/NASA funded project. It is an 8legged walking machine with exceptional outdoor and rough terrain mobility. Figure 1 shows a picture of the system. The robot is controlled using a biomimetic approach of ambulation control. The approach is based on two biological control primitives: Central Pattern Generators and Reflexes. Using this approach, omni-directional walking and smooth gait transitions can be achieved. Additionally, the posture of the robot can be changed while walking. The robot was successfully tested in rough terrain with obstacles as high as the robot's body and in different terrains such as sand, grass, concrete and rock piles. Below we address its major components.

\section{A. Legs}

The current leg design (Fig. 3) provides 3 degrees of freedom, which is the minimum needed for a robust, outdoor walking robot, e.g. it provides the possibility to walk in an omni-directional fashion in narrow environments. The leg consists of a thoracic joint for protraction and retraction, a basalar joint for elevation and depression and a distal joint for extension and flexion of the leg. The joints are actuated by standard 6 Watt $24 \mathrm{~V}$ DC-Motors with a high gear ratio for sufficient lifting capacity.

An important constraint in the development of the legs was their outdoor capability. Therefore a good tradeoff between making the leg as light as possible to improve its lifting capacity and shielding it against the environmental influences like dust and water had to be found. We achieved a weight of 850 grams and a weight to lifting-capacity ratio of 1:8. This is a prerequisite to walk up steep rises or to walk over obstacles higher than the robot itself. Another important feature are the compliant elements integrated in the design which make the robot robust enough to withstand the mechanical stresses in outdoor/rough terrain. 

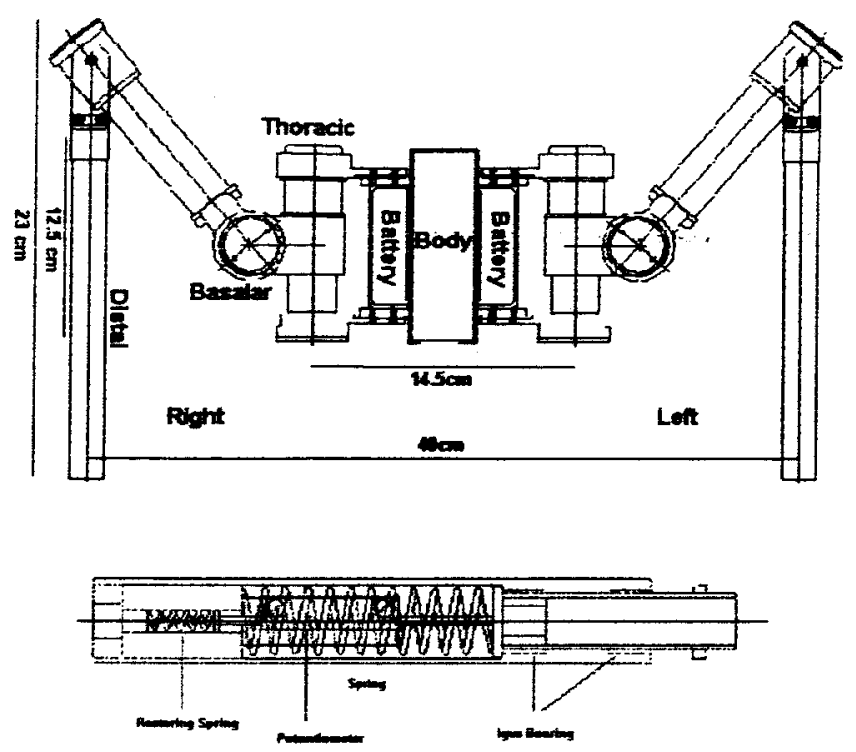

Figure 3. The mechanical design of the original Scorpion legs.

The front view of the robot shows left and right side legs with the body in the center. Each leg consists of 3 parts: 1) thoracic joint, 2) basalar joint and 3) distal joint. The distal segment contains a spring damped compliant element with a built in potentiometer to measure contact and load on individual legs.

\section{B. Sensors}

The robot is equipped with the following proprioceptive sensors:

Motor encoders for each motor to measure the relative joint angle

Hall-Effect Motor Current Sensors for each motor

Analog load/pressure sensors in each foot tip

Power management sensors, providing current battery voltage and current power drain

Three-dimensional inclinometers (pitch, roll and yaw)

The following exteroceptive sensors are integrated:

Ultrasound distance sensor for obstacle avoidance

Compass sensor for heading control

Contact/pressure sensors at the foot tip

Stereo CCD Camera system

It is important to note that the legs itself can be used as an exteroceptive sensors. One can use the current sensors of the joint motors during the movement as a tactile sensor during movement. In order to allow an operator to communicate with the robot or to take data samples during a test run, the robot is currently equipped with an wireless $28 \mathrm{~K}$ Baud bi-directional communication link and a PAL CCD Camera with a $5 \mathrm{GHz}$ video/audio link for video transmission.

\section{Electronics}

The following section describes the electronic subsystem currently integrated in the scorpion robot. The electronic subsystem is currently designed to independently control 32 of the servo motors described above. The hardware centers on the powerful Motorola MPC 555 microcontroller and an XILINX FPGA, for low level actuator control. 


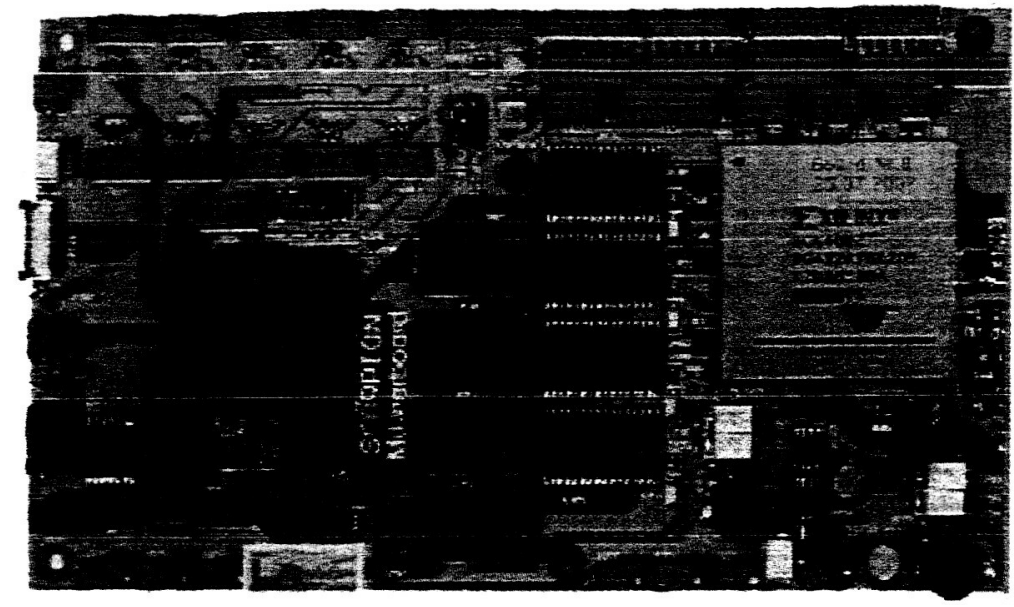

Figure 4. The electronic subsystem is capable of controlling 32 servomotors independently without requiring the main CPU to interfere, thus leaving resources available for higher-level processes.

The main features of the electronic subsystem are listed below:

Two programmable units:

MPC 555

Xilinx FPGA

Peripheral highlights:

$8 \mathrm{MB}$ Flash EEPROM, $4 \mathrm{MB}$ SRAM

32 DC Motor drivers (5A max.) (Wired to the FPGA)

32 on board Current Sensors

32 AD Ports (on additional board) wired to the FPGA

DECT Wireless Link Module Connector

Different Voltage Supplies: $1.8 \mathrm{~V}, 3.3 \mathrm{~V}, 5 \mathrm{~V}, 15 \mathrm{~V}$ on board

12 to 36 Volts Operating Voltage

The MPC555 controller is comprised of the following features:

High performance 32-bit RISC PowerPC Core with FPL

Embedded Flash (448k)

32 bit data bus, 24 bit address bus with 4 Chip Selects (used to comm. With: 8 MB Flash, 2 x 2 MB RAM \& the FPGA)

MIOS (2 parallel ports, 8 PWM, 10 Capture/Compare Units, Counter...)

$2 \times$ Programmable TPU ( 32 channels)

$2 \times$ CAN, $3 \times$ serial ports

Dual Queued A/D-Converter (up to 81 channels multiplexed)

Temperature range: $-40^{\circ}$ to $125^{\circ}$

BDM-Port

Development Tool: Metrowerks Code warrior for Embedded Systems 5.0

Programming languages: C \& Assembler

The FPGA is comprised of the following features:

Type: Virtex E XCV400E (432 pins)

Development Tools: Xilinx ISE 4.1 with FPGA Express 3.6

Programming Language: VHDL (Very High Speed Integrated Circuit

Description Language + Predefined Xilinx-Cores

Clock: $20 \mathrm{Mhz}$ clock (Max.clock cycle in this design is $40 \mathrm{MHz}$ )

Select Map Mode Compatible 
The electronic subsystem is designed to allow for fast and smooth control of up to 32 servo and/or DC motors. The separation of the FPGA for low level actuator control and the MPC555 for behavior level control was implemented in order to be able to run the low level PID controllers at a maximum speed. In the presented design, the PID controllers operate at up to $20 \mathrm{khz}$ for each individual motor channel. The FPGA receives position information for each motor/joint from the MPC555 and computes the appropriate PWM (pulse width modulated signals) to drive the motor to the destination. A PID controller, also implemented in the FPGA assures the correct positioning and holding of that position. Because of the great speed of the PID controllers we can smoothiy drive the motors over their performance range.

\section{Ambulation Control}

The Scorpion's locomotion control architecture ${ }^{3}$ is a combination of two seemingly contradictory biological approaches: the Central Pattern Generator (CPG) model and the pure reflex driven approach. ${ }^{4,5,6}$

A CPG is able to produce a rhythmic motor pattern even in the complete absence of sensory feedback. The general model of a CPG has been identified in nearly every biological species even though the specific instantiations vary among the species to reflect the individual kinematic characteristics in the animals. This idea has shown to be promising as a concept to stabilize locomotion in kinematically complex robotic systems as it resembles the divide and conquer strategies that are reflected in nearly all solutions to complex control problems. ${ }^{7}$

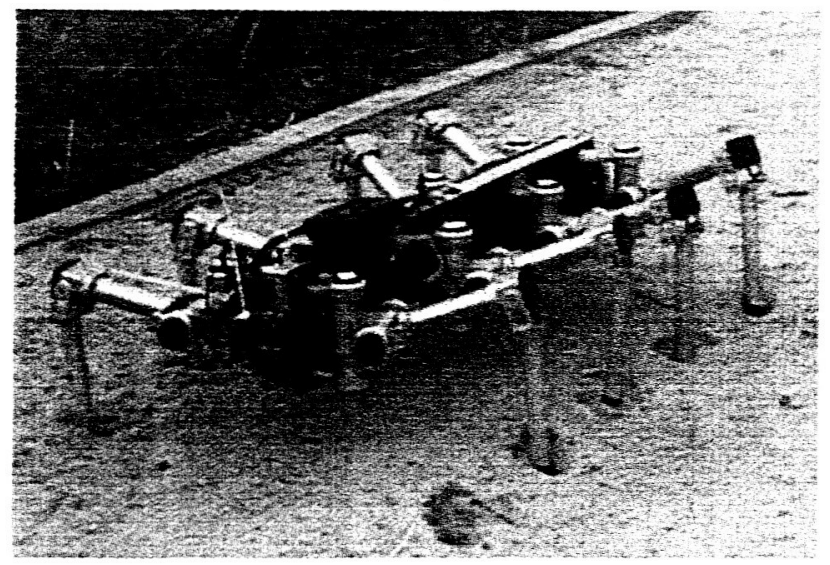

Figure 5. The Scorpion robot during an autonomous exploration in a sand bed.

The robot's feet penetrated the sand to a depth of approximately $3-5 \mathrm{~cm}$. A reflex mechanism helped to prevent the feet from getting stuck.

The reflex based control approach can also be observed in the animal kingdom ${ }^{6}$. A reflex can be viewed as a closed loop control system with fixed input/output characteristics. In some animals, like the locust, this concept is said to actually perform all of the locomotion control and no further levels of control, like the CPG, are involved ${ }^{5}$. Whether or not complex motion control can be achieved only via reflex systems is subject to further discussion, however, the concept of a set of fixed wired reactions to sensory stimuli is of high interest to robotics developers who aim to gain stability in the system's locomotion. The design of the control architecture described here was thus driven by these two concepts. The CPG approach appeared to be useful in generating rhythmic walking patterns that could be implemented in a computation-efficient manner, while the reflex driven approach seemed to provide a simple way to stabilize these walking patterns by providing 1) a set of fixed situation-reaction rules to external disturbances and 2) as a way to bias leg coordination among multiple independent legs ${ }^{5}$. Figure 6 outlines the general idea. 


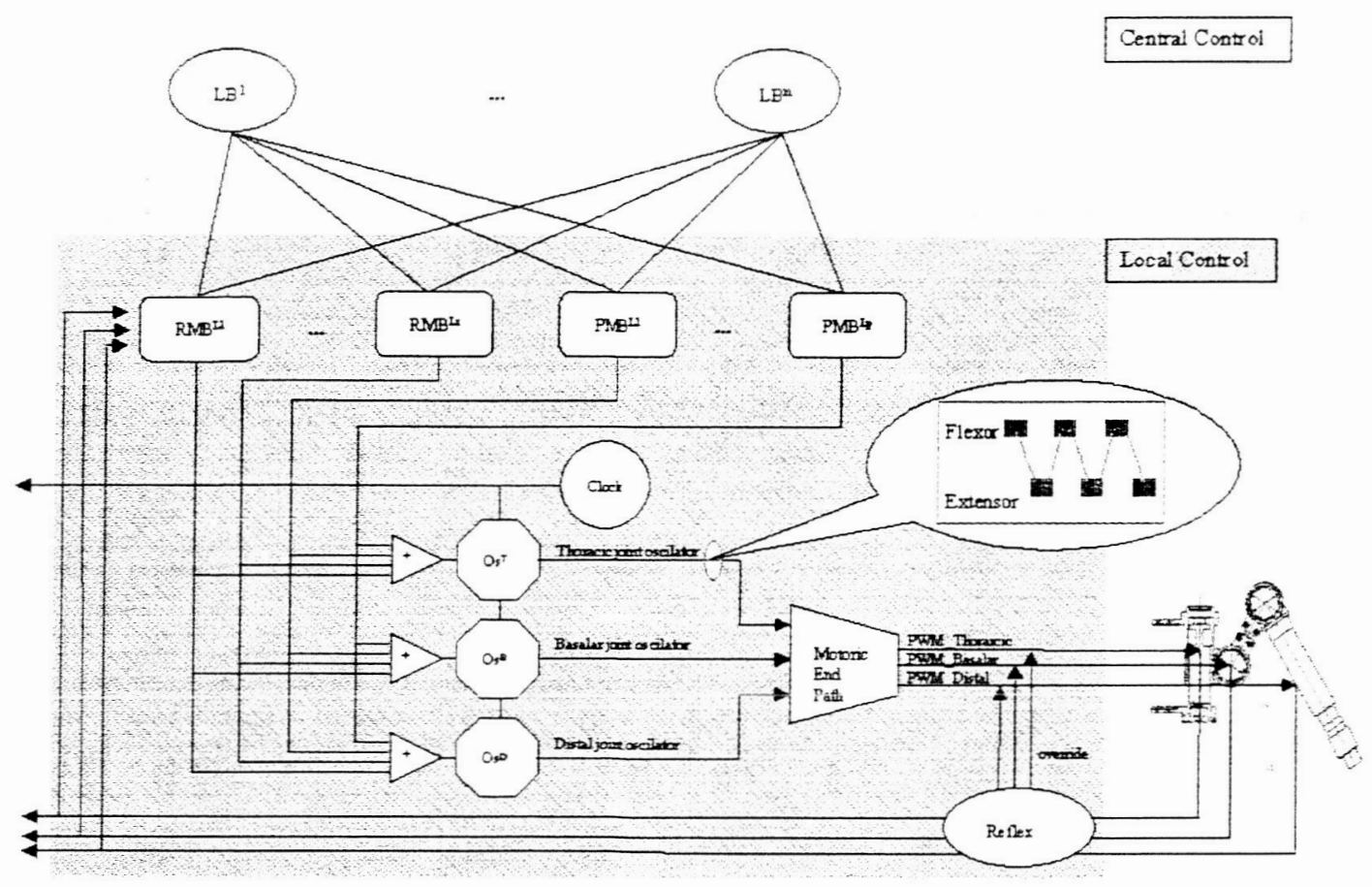

Figure 6. The overall architecture for low level actuation.

On the global level (upper light gray area) we have implemented the Locomotion Behaviors (LB's), typically (Forward, Backward and Lateral locomotion). These global behaviors are connected to all local leg controllers and activate (with continuous strength) the local (per leg) motion behaviors. At the same time they implement the interleg phase relation by setting/resetting the local clocks. The local level (dark gray area) implements Rhythmic Motion Behaviors (RMB's) and Postural Motion Behaviors (PMB's). These behaviors simultaneously influence the amplitude and frequency parameters of three oscillating networks $\left(O S^{\mathrm{T}}, \operatorname{OS}^{\mathrm{B}}\right.$ and $\left.O S^{\mathrm{D}}\right)$. The oscillators are connected to a common clock which is used for local and global (in relation to other legs) synchronization purposes. The oscillators output is a rhythmic, alternating flexor and extensor stimulation signal (see callout box in Figure 6) which is implemented as splined sine waves. This activation signal represents the desired behavioral locomotion, which is translated into PWM signals via the motor end path. Inline with the output of the motor end path are a set of perturbation specific reflexes, which are implemented as 'watchdogs'. They override the signals on the end path with precompiled activation signals if the sensor information from the physical joints meets a set of defined criteria.

\section{A. Low Level Locomotion Control}

This approach was implemented using inter-leg coordination data as observed in real scorpions ${ }^{8}$ and successfully tested on our 'SCORPION' robot study as shown in Figure 7. Parts A through C of Figure 7 illustrates the data for the performance of one leg. The solid line is the real angle of the leg, measured with the motor encoders. The angles for the distal and the basalar joints increase during elevation, while the angle for the thoracic joint increases during protraction. The frequency was set to $1.3 \mathrm{~Hz}$ (19 time units on the $\mathrm{x}$-axis). The data was taken every $1 / 25$ of a second and the curves are directly computed from the raw data. The mean starting position is at 37 degrees for the thoracic joint, 121 degrees for the basilar joint, and 30 degrees for the distal joint. At first only a local FORWARD behavior is stimulated (until $t=1375$ ), then the LATERAL behavior is activated simultaneously. Because of the equal strength of the activation the system now tries to walk forward as well as laterally, which results in a diagonal walking direction. At approximately time $t=1460$ the activation of the FORWARD behavior is set to 0 which leaves only the LATERAL behavior to influence the oscillator networks. Thus the system walks laterally, which can be observed from the data as the amplitude of the thoracic joint is 0 while the basalar and especially the distal joint perform large amplitude oscillations. Subsequently the described process is reversed.

6

American Institute of Aeronautics and Astronautics 
A

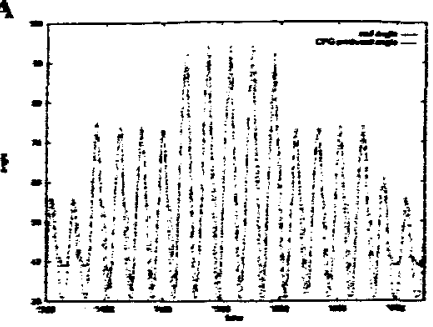

$\mathbf{B}$

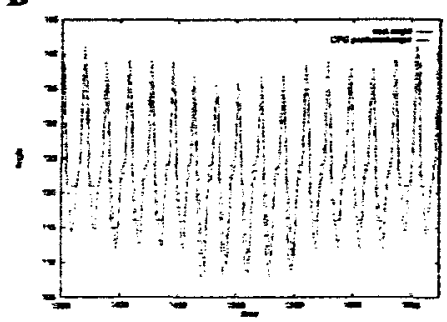

C

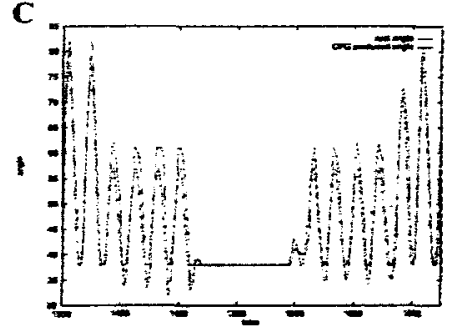

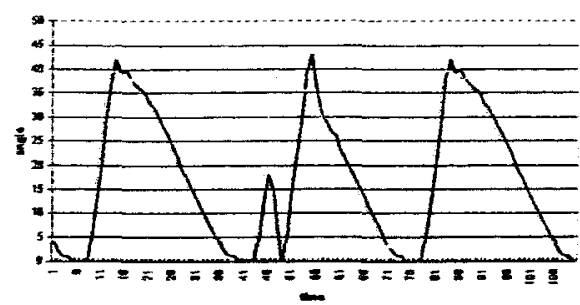
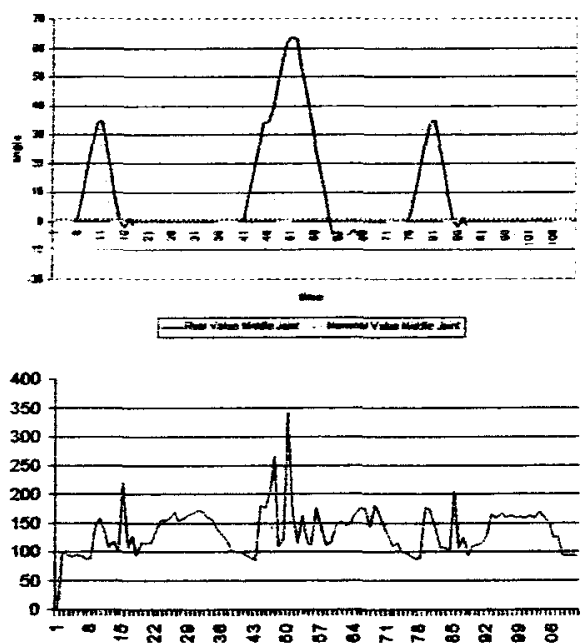

Figure 7. Reflex action

Traces $A$ through $C$ show the movements of the thoracic (A), the basalar (B) and the distal (C) joints during a transition from forward walking (pure FORWARD activation), to diagonal (equal activation of FORWARD and LATERAL), to lateral walking (pure LATERAL activation) and back to forward walking. See Figure 6 for the pathways of activation. A reflex initiated at a leg during a course through a rock bed. The current in the thoracic joint (Trace $C$ ) increased as a result of the obstacle blocking the way. At the same time the angular displacement error (Trace A) in the thoracic joint increased, indicating an exception in the regular swing cycle As a result of these factors the basalar joint controller initiated the reflex (Trace B) that elevated the leg further, thereby overcoming the obstacle.

The current bio inspired locomotion control concept is based on Central Pattern Generators (CPG) and pure reflexes. This simple approach will be made much more robust by the inclusion of force control .To achieve a high level of biological stability in the Scorpion Robot, the dynamic controller will explicitly control the angular momentum of the machine for standing, walking and running maneuvers. As the robot moves through space, a desired center of mass trajectory and impedance field will guide stance-leg joint torques and stiffness. By regulating angular momentum ${ }^{9}$ a desired center of pressure (CP) trajectory will be derived giving the current $\mathrm{CM}$ positions and forces, and that $\mathrm{CP}$ trajectory will define swing-limb dynamics and ground-foot placement. The above bio inspired controllers will be embedded in a behavioral architecture. We plan to implement complex locomotion/manipulation behaviors. Simple instantiations such as Forward, Backward and Lateral locomotion have already been implemented. We will develop more sophisticated examples like grasping and climbing within the same behavioral architecture framework. These global behaviors modulate the parameters of the individual leg , local controllers' and enable the system to adapt its walking strategy to varying terrains., In combination with the inherent stability produced by the force control approach these behaviors allow for stable, robust execution of locomotion over 
varying rough terrain with smooth transitions between walking, scampering over rocks and climbing. Finally, the force control approach will enable locomotion behaviors to adapt to steep surfaces in rappelling mode.

The behavioral level also implements the interface to the high level planning implemented mainly on the rover system. We plan to directly interface with the planning algorithms already in place on K9. Behaviors on the scout can be activated by the rover system in an open or closed loop fashion.

Another purpose of the behavioral level is the implementation of perception behaviors. These behaviors are activated by the scout itself and can be local or remote, where the remote perception behavior actually triggers sensor data acquisition on the rover using its more sophisticated sensor capabilities. These data will be preprocessed and transferred to the scout in a common data interchange format. Local perception behaviors will involve passive sensor acquisition using onboard sensors like ultrasound, infrared etc but also incorporate behavioral acts using the robots appendages (Legs). For instance these could be used for probing (pushing against, or pulling) environmental features, e.g. a possible stone may be just a pile of sand. The data preprocessed in this way can then be transferred back to the rover for further global decision making, e.g. on path planning. Mechanical modifications like grippers, mounting sites for special sensors and a rappelling mechanism will be developed as well.

\section{The Robotic team control problem}

The robot team shall be evaluated on simulated scientific investigations of Martian-like sites on Earth (Ames test site) that are difficult or impossible to access with more conventional wheeled rovers. Primarily, we intend to focus on maneuvering on the faces of steep cliffs and canyon walls. These are sites that are believed to be of highest scientific interest on Mars. This is due to the fact that in general cliff faces reveal the geological history of a site, but, for Mars, there is the additional indication of probable water seepages that appears to have occurred in the relatively recent past on several cliff sides, as revealed by recent satellite images. One of the main challenges of this approach is to combine a tough control problem, robust low level locomotion on rough, difficult terrain, with high level planning, execution, and execution control algorithms on two physically separated and kinematically different robotic systems. In the team oriented approach presented here, the work will focus on the 8-legged robot ,Scorpion' by implementing a biologically inspired, set of complex motor skills (locomotion and manipulation), that are embedded in a behavior based architecture. The high level planning, execution and execution control algorithms will be implemented on the wheeled rover system. By distributing the autonomy and intelligence among 2 physically distinct and kinematically different systems, we believe we will be able to achieve a greater amount of overall autonomy and intelligence for the robot team.

A robust bidirectional communication link between the rover and the legged scout robot needs to be developed. The communication link will be used for 2 purposes. A) a selection protocol of behaviors. The protocol serves to select behaviors on the scout system as well as to report termination conditions to the rover system. B) to exchange sensor information for local action selection on the scout as well as global planning on the rover. Sensor data will be acquired on both systems. They will be processed locally and translated into a common information exchange format, transmitted to the team robot and then used for further decision making.

We believe that our concept of bio inspired ambulation control on the low-level side of the problem will help to optimize the high level planning and execution end of the problem by providing a robust, behavior based set of low level action primitives. On the other hand the ambulation control problem will be easier to tackle if high level planning information (e.g. bird's eye views on the immediate local terrain to be traversed) can be incorporated on the low level locomotion side.

\section{Conclusions}

We have implemented a robust locomotion control concept. The concept is behavior based and incorporates biologically inspired mechanisms of ambulation control for locomotion on rough, changing and partially known or unknown substrates. The behavior based layer will allow us to define a suitable interface to high level planning and execution algorithms, while the bio-inspired low level locomotion control will allow us to implement robust locomotion behaviors on difficult terrain. This feature will enhance the reliability of the behavioral level dramatically and thereby increase the level of stability in execution loops on the high level system. 


\section{Acknowledgments}

Initial work on the Scorpion was made possible by DARPA (N00014-99-1-0483). More recent funding was provided by NASA (USRA, 8008-003-002-01) and from seed funding at Ames and at the University of Bremen. Future development will be pursued in the context of international agreements with ESA and DLR.

\section{References}

${ }^{1}$ Kirchner, F., Spenneberg, D., Linnemann, R. :A biologically inspired approach towards robust real world locomotion in legged robots, In book: Neurotechnology for Biomimetic Robots, Ayers, J., Davis, J., Rudolph, A., (eds.), MT-Press, MA, USA, 2002

${ }^{2}$ Pirjanian, P., Leger,C.,Mumm,E.,Kennedy,B., Garrett, M, Aghazarian, Schenker, P.S. and Farritor, S. „Distributed Control for a Modular, Reconfigurable Cliff Robot" in Proc. To to 2002 International Conf on Robotics and Automation (ICRA2002), Wash DC, May 2002.

${ }^{3}$ Spenneberg, D, and Kirchner, F.: Omnidirectional Walking in an Eight Legged Robot, Proc. International Symposium of Robotics and Automation (ISRA2000), Monterrey, Mexico; 2000

${ }^{4}$ Ayers, J., Kashin, S., Blidberg, D.R., and Massa, D.: Biologically-Based Underwater Robots in Unmanned Systems, vol. $12,30-36 ; 1994$

${ }^{5}$ Cruse, H. et al.: Walknet- a biologically inspired network to control six-legged walking; Neural Networks. 11, 1435$1447 ; 1998$

${ }^{6}$ Delcomyn, F.: Neural Basis of Rhythmic Behavior in Animals; in: Science, vol. 210, 492-498; 1980.

${ }^{7}$ Brooks, R A.: A robust layered control system for a mobile robot; in IEEE Joumal of Robotics and Automation, vol. 2, $14-23 ; 1986$.

${ }^{8}$ Bowerman, R. F.: The Control of Walking in the Scorpion, I. Leg Movement during Normal Walking, J. comp. Physiol., Vol. 100, 183-196, 1975.

${ }^{9}$ Popovic M., Herr H. (2002). Conservation of Angular Momentum in Human Movement. MTT AI Lab Abstracts. 